\title{
Psychological Stress Alters Ultrastructure and Energy Metabolism of Masticatory Muscle in Rats
}

\author{
Yong-Jin Chen, Fei Huang, Min Zhang, and Hai-Yan Shang \\ Department of General Dentistry and Emergency, School of Stomatology, The Fourth Military Medical University, \\ Xi'an, Shanxi 710032, China \\ Correspondence should be addressed to Yong-Jin Chen, cyj1229@fmmu.edu.cn and Min Zhang, zhangmin@fmmu.edu.cn
}

Received 1 June 2010; Revised 14 September 2010; Accepted 16 September 2010

Academic Editor: M. Smith

Copyright ( $(2010$ Yong-Jin Chen et al. This is an open access article distributed under the Creative Commons Attribution License, which permits unrestricted use, distribution, and reproduction in any medium, provided the original work is properly cited.

To investigate the effects of psychological stress on the masticatory muscles of rats, a communication box was applied to induce the psychological stress (PS) in rats. The successful establishment of psychological stimulation was confirmed by elevated serum levels of adrenocorticotropic hormone (ACTH) and changed behaviors in the elevated plusmaze apparatus. The energy metabolism of the bilateral masseter muscles was tested via chemocolorimetric analysis, whereas muscle ultrastructure was assessed by electron microscopy. In comparison to the control group, the PS group showed evidence of swollen mitochondria with cristae loss and reduced matrix density in the masticatory muscles after three weeks of stimulation; after five weeks of stimulation, severe vacuolar changes to the mitochondria were observed. Increased vascular permeability of the masticatory muscle capillaries was found in the five-week PS rats. In addition, there was decreased activity of $\mathrm{Na}^{+}-\mathrm{K}^{+}$ATPase and $\mathrm{Ca}^{2+}$-ATPase and a simultaneous increase in the activity of lactate dehydrogenase and lactic acid in the masticatory muscles of PS rats. Together, these results indicate that psychological stress induces alterations in the ultrastructure and energy metabolism of masticatory muscles in rats.

\section{Introduction}

A number of studies have examined the role of psychological stress in the development of masticatory muscle disorders (MMD). War-related psychological stress, the performance of mental arithmetic, and the solving of five-letter anagrams can increase masticatory muscle activity and are believed to be associated with MMD [1-3]. Moreover, psychological tension can induce muscle fatigue and spasm, resulting in MMD [4-6]. A relationship between stress and MMD has been reported in children, adolescents, and adults [7-9]. Additionally, most patients with MMD experience moderate to severe depression or anxiety $[10,11]$. On the other hand, cognitive-behavioral [12], habit reversal [13], and biobehavioral treatment [14] strategies are all known to be beneficial in the management of MMD, thus reflecting another line of evidence demonstrating a relationship between psychological stress and MMD.

All of the studies conducted to date illustrate the importance of psychological factors in $\operatorname{MMD}[15,16]$. However, it is not yet possible to determine how the mental/ psychological factors affect the muscle and even to facilitate the MMD. Clinical studies using traditional electromyography (EMG) to assess the role of psychological stress in MMD have shown significantly increased EMG activity of masticatory muscles under stress conditions [3, 17-19]. Similar results have been observed in animals $[20,21]$. Under the emotional stress, changes in the masticatory behaviour and hormones such as glucocorticoids were accompanied by a modification of particular $\mathrm{MyHC}$ isoforms of the muscle, which showed evident faster phenotype due to a higher percentage of either pure MyHC-2b containing fibres or hybrid MyHC-2x/d and -2b expressing fibres [22]. Furthermore, a decrease in oxygen saturation of muscle blood around the onset of mental stress proved the potential role for psychological stress in hemodynamic alterations, which might occur via their regulation by the sympathetic nervous system (SNS) [23, 24]. An elevation of histidine decarboxylase activity in skeletal muscles during stress was induced, partly or wholly by muscle activity and/or muscle tension [25]. However, muscular mechanism underlying emotional stress is not well understood and appears to be equally complex. 
The hypothesis of this paper was that psychological stress could alter the ultrastructure and energy metabolism of masticatory muscles. Using an emotional stress paradigm, which employed the use of intraspecies emotional communication within a communication box [8], we introduced experimental correlates of psychological stress, such as anxiety and depression, in rats and then tested our hypothesis on their masseter muscles. The masseter muscle is useful in this type of research because there are large amounts of spindles in these muscles [26], which are critical in determining muscle contraction and mandibular movement in response to stimulation [27]. This study sought to assess alterations in the ultrastructure and energy metabolism of masticatory muscles of rats under psychological stress.

\section{Materials and Methods}

2.1. Experimental Design. Forty-eight male Wistar albino rats weighing between 160 and $180 \mathrm{~g}$ (approx. 35 days old) were housed in $80 \mathrm{~cm} \times 45 \mathrm{~cm} \times 40 \mathrm{~cm}$ cages in a temperature-controlled room at $24^{\circ} \mathrm{C}$ under a 12 -hour light/dark cycle and were given free access to food and water. Before experiments, they were housed in a communication box one hour a day for five days to allow them to acclimatize to the box. The rats were then randomly divided into control (CON), foot-shocked (FS), and psychological stress (PS) groups, with each group having 16 rats. FS and PS rats were housed in one communication box during the procedure as described below, with the FS rats receiving electric foot shock and the PS rats being subjected to psychological stress. The rats in the control group were housed in another communication box under the same conditions but were not subjected to electric foot shock or psychological stress. The same study was performed twice, and the durations of psychological stimulation were three weeks and five weeks. Thus, 24 animals were used per study, with eight animals in each of the three groups.

After three or five weeks of stimulation, the behaviors of the eight rats from the control or PS group were immediately evaluated by an elevated plus-maze apparatus. Blood was drawn from the ophthalmic artery, and serum samples were prepared for the measurement of serum stress indices. The rats were then sacrificed by intraperitoneal injection of an overdose of thiamylal sodium, and their bilateral deep masticatory muscles were removed. All muscle samples were obtained according to guidelines established by the University Internal Review Board for use of Mice Subjects. The experimental procedures were reviewed and approved by the Ethics Committee of the Fourth Military Medical University. The middle bellies of each muscle sample were dissected for ultrastructure observation by electron microscopy.

2.2. Animal Model for Psychological Stress. The communication box (16) was selected as the psychological stress apparatus in this study. It consisted of 16 compartments that were $16 \times 16 \mathrm{~cm}$ each and were separated by transparent plastic boards with several small holes. The boards prevented

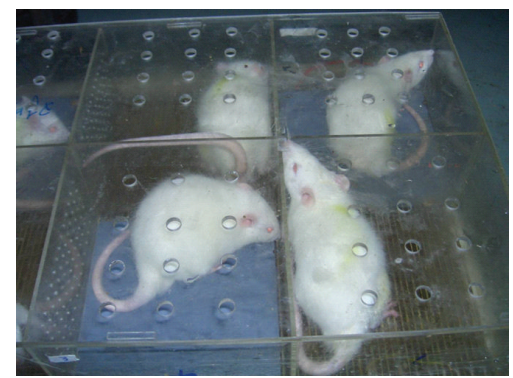

(a)

\begin{tabular}{|c|c|c|c|}
\hline PS & FS & PS & FS \\
\hline FS & PS & FS & PS \\
\hline PS & FS & PS & FS \\
\hline FS & PS & FS & PS \\
\hline
\end{tabular}

(b)

FIgURE 1: The communication box used in the experiment. (a) Photos of the communication box with rats inside. The grid floors of the psychological compartment were covered by blue plastic plates. (b) Schematic diagram of the communication box. FS refers to the foot-shocked group, and PS refers to the psychological stress group.

the animals from making physical contact with each other but allowed them to receive cues, such as visual, auditory, and olfactory sensations, from the neighboring animals. Each compartment was equipped with a grid floor of stainless steel rods, which were $5 \mathrm{~mm}$ in diameter and placed at intervals of $0.3 \mathrm{~cm}$. A $48-\mathrm{V}$ electric generator, which was made by the Department of Biomedical Engineering of the Fourth Military Medical University, was connected to the grid floor to produce an electric current and generate an electric foot shock every two seconds. The grid floors of eight of the compartments were covered by plastic plates to prevent electric foot shock and served as non-foot-shock compartments for the PS rats (Figure 1).

Stress stimulation within the communication box commenced five days after electrode installation. Prior to the day of stress stimulation, the PS rats, together with the FS rats, were individually confined in each compartment of the communication box for one hour per day without any electric foot shock for one week to allow them to acclimatize to the surroundings. The electric foot shock was then introduced to the FS rats (stress senders) for one hour a day at a fixed time in the morning. The PS rats (stress responders) confined in the non-foot-shock compartments were simultaneously exposed to emotional cues from the neighboring FS rats, such as shrieks, smells of urine or feces, and jumping responses. Consequently, the PS rats were assumed to be in a state of fear or anxiety. The purpose of this study was to explore the effects of the pure 
psychological stress upon the masseter muscles. But the footshocked group rats experienced a great part of physical stress, so we did not include this group in the experimental evaluations. The foot-shocked group was only used to induce psychological stress in the neighboring PS rats, and they were not included in the following investigations.

2.3. Behavioral Evaluation. The elevated plus-maze (EPM) apparatus (RD1208, Shanghai Mobiledatum Corporation, Shanghai, China) [28] consisted of two open arms $(50 \times$ $10 \mathrm{~cm})$ and two enclosed arms $(50 \times 10 \mathrm{~cm}$, with $50-\mathrm{cm}$ high walls) extending from a central square platform (10 $\times 10 \mathrm{~cm}$ ). The same types of arm were arranged in the opposite position. The apparatus was elevated $50 \mathrm{~cm}$ above the floor in a quiet room with temperature controlled at $20^{\circ} \mathrm{C}$, with the light just bright enough to clearly observe the rats movement within a 1.5 -meter range. Two of the opposing arms $(50 \mathrm{~cm} \times 10 \mathrm{~cm})$ were enclosed by $40-\mathrm{cm}$ high side and end walls (enclosed arms), the other two arms were not installed with walls (open arms). In the beginning, animals were placed in the central area $(10 \times 10 \mathrm{~cm})$ of the maze, facing an enclosed arm. The exposure lasted for five minutes, and certain parameters were recorded. Entry into one arm was recorded when an animal placed all four paws past the line dividing the central square from the open arms. The test arena was wiped with a damp cloth after each trial. The number of entries into the open/closed arms and the time spent in open arms/closed arms were measured by an observer blind to the treatment conditions of the animals. Then, the percent of open arm entries $(100 \times$ open/open + enclosed entries, $\mathrm{OE} \%$ ) and time spent in the open arms (100 $\times$ open/open + enclosed arm time, OT\%) of the EPM were calculated for each rat. We used $\mathrm{OE} \%$ and OT\% to analyze the anxiety level of each animal [29-32].

2.4. Serum Assay. The serum concentrations of cortisol and adrenocorticotropic hormone (ACTH), stress indices, were measured by radioimmunological analysis kits (Northern Bioengineering Institute, Beijing, China) according to the protocols provided by the manufacturer.

2.5. Ultrastructure. The middle bellies of the bilateral deep masseter muscles were dissected and cut into $1-\mathrm{mm}$ pieces and then fixed with $4 \%$ glutaraldehyde and $1 \%$ osmic acid. The samples were embedded in Epon812, sectioned by LKBV ultramicrotome (LKB, Bromma, Sweden), and stained with uranyl acetate and lead citrate. The ultrastructures of the muscles were then observed with transmission electron microscopy (JEM-100SX, JEOL Company, Japan).

2.6. Energy Metabolism. A sample of masticatory muscle $(0.2 \mathrm{~g})$ was minced and homogenized with $1.8 \mathrm{~mL}$ distilled water. Then, $0.1 \mathrm{~mL}$ homogenates were diluted 10 -fold with water. After incubation for $15 \mathrm{~min}$ at room temperature, the mixtures were then centrifuged for $10 \mathrm{~min}$ at $3000 \mathrm{r} / \mathrm{min}$. The supernatants were transferred into new tubes and stored at $4^{\circ} \mathrm{C}$ before further examination. The activities of $\mathrm{Na}^{+}$$\mathrm{K}^{+}$ATPase, $\mathrm{Ca}^{2+}$-ATPase and lactate dehydrogenase (LDH) and the contents of lactic acid (LD) were measured by commercial kits according to the protocols provided by the manufacturer (A070-5, A019-2, A020, Nanjing Jiancheng Bioengineering Institute, Nanjing, China) using a UV-754 spectrophotometer (Shanghai Third Analytical Instrument Factory, Shanghai, China). The protein concentrations were measured via the Bradford method, with BSA as the standard [33]. The substrate for ATPases is ATP, which is split by the ATPase into ADP and inorganic phosphorus (Pi). Determination of the amount of Pi indicates the level of ATPase activity. Units of ATPase activity were shown as the content of Pi generated per hour (h) by decomposition of protein per gram $(\mathrm{g})$, and the total protein content was determined by Lowry method. Therefore, the amount of inorganic phosphorus was used to detect the activity of $\mathrm{Na}^{+}-\mathrm{K}^{+}$ATPase and $\mathrm{Ca}^{2+}$-ATPase at the wavelength of $636 \mathrm{~nm}$. With regard to $\mathrm{LDH}$, it catalyzes the reaction that generates pyruvic acid from lactic acid; pyruvic acid can then react with 2,4-dinitrophenylhydrazine to produce pyruvate dinitrophenylhydrazone, which is brownish red in alkaline solutions. Enzyme activity can be calculated through colorimetry; the detecting wavelength is $440 \mathrm{~nm}$. Further, by catalytic dehydrogenation of LDH, lactic acid changes into pyruvate and NAD+ changes into NADH. By dehydrogenation of PMS, NBT deoxygenizes purple-colored objects; the relationship between this change and LD is linear and occurs at an absorbance of $530 \mathrm{~nm}$. Therefore, the wavelength for $\mathrm{Na}^{+}-\mathrm{K}^{+}, \mathrm{Ca}^{2+}$-ATPase detection LDH detection, and LD detection was $636 \mathrm{~nm}, 440 \mathrm{~nm}$, and $530 \mathrm{~nm}$, respectively. The enzyme activities were expressed as the incorporation of total phosphate into the substrate per $\mathrm{mg}$ protein per hour. In this part, we stored the supernatants for no more than 6 hours at $4^{\circ} \mathrm{C}$ and finished the detection of all samples in one day.

2.7. Statistical Analysis. Experimental data were analyzed by one-way analysis of variance (ANOVA) across the control group, the three-week PS group, and the five-week PS group using SPSS, version 11.0 (SPSS Co., Chicago, Illinois). The SNK-q test was further used to calculate any differences between the two groups. A $P$ value less than .05 was considered statistically significant.

\section{Results}

To confirm that the experimental rats were in a state of stress, we first analyzed the serum levels of cortisol and ACTH in the rats. As shown in Figure 2(a), the concentrations of cortisol were $12.46 \pm 2.67 \mathrm{ng} / \mathrm{ml}$ and $11.78 \pm 2.35 \mathrm{ng} / \mathrm{ml}$ in the PS group after three or five weeks of psychological stimulation, respectively. These values were markedly increased compared with those of the control group $(6.19 \pm 1.47 \mathrm{ng} / \mathrm{ml}$ and 5.93 $\pm 1.54 \mathrm{ng} / \mathrm{ml}$, resp., $P<.05)$. Similar changes were observed in ACTH levels. As shown in Figure 2(b), in the PS group, the serum ACTH concentrations were $25.46 \pm 5.12 \mathrm{ng} / \mathrm{ml}$ and $23.49 \pm 4.95 \mathrm{ng} / \mathrm{ml}$ in rats after three and five weeks of stimulation. By contrast, during the same periods, the serum concentrations of ACTH in the control group were 


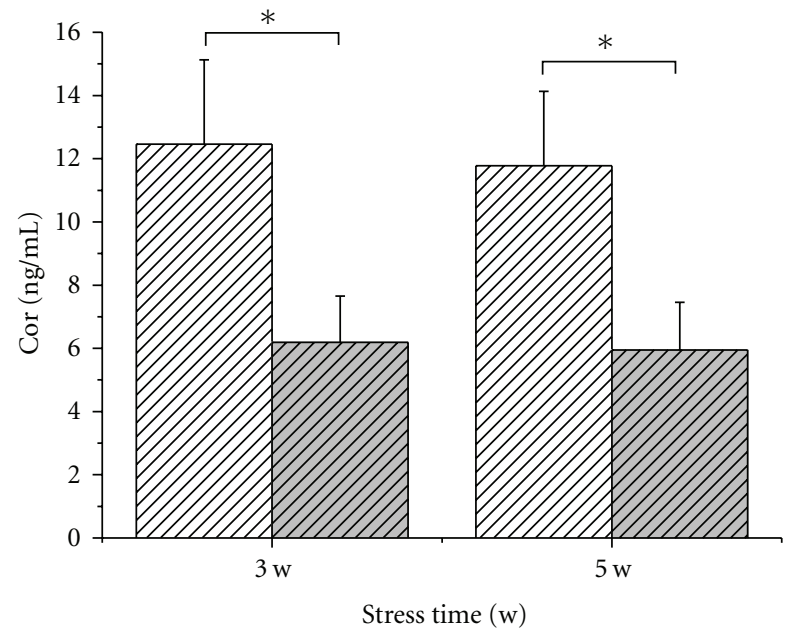

VZT PS group EIID CON group

(a)

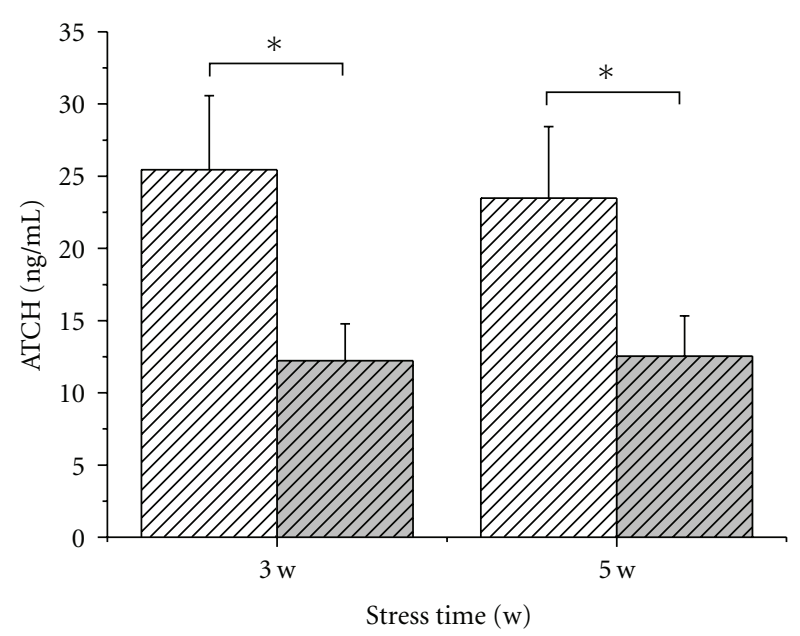

ZIJ PS group
ZIJ CON group

(b)

FIGURE 2: The levels of cortisol and adrenocorticotropic hormone in serum. Shown are the levels of cortisol (Cor, in (a)) and adrenocorticotropic hormone $(\mathrm{ACTH}$, in (b)) in the serum of rats in the psychological group (PS) and control group (CON). Data were expressed as $\bar{X} \pm \mathrm{SE}$ and analyzed in eight rats from each group $\left({ }^{*} P<.05\right.$, PS group versus CON group).

only $12.22 \pm 2.56 \mathrm{ng} / \mathrm{ml}$ and $12.54 \pm 2.79 \mathrm{ng} / \mathrm{ml}$, which were significantly lower than those in the PS group $(P<.05)$, indicating the rats in PS group were anxious.

We then compared the behaviors of rats after three or five weeks of psychological stimulation with those of the control group by EPM tests. As shown in Figure 3(a), percentages (\%) of entry into open arms (open arm entry, OE) in the PS rats were $34.02 \pm 2.89 \%$ and $24.66 \pm 1.99 \%$ after three or five weeks of stimulation, respectively, while those of control group were $48.94 \pm 2.94 \%$ and $48.65 \pm 3.81 \%$, respectively, which were significantly higher than the PS groups. Similarly,

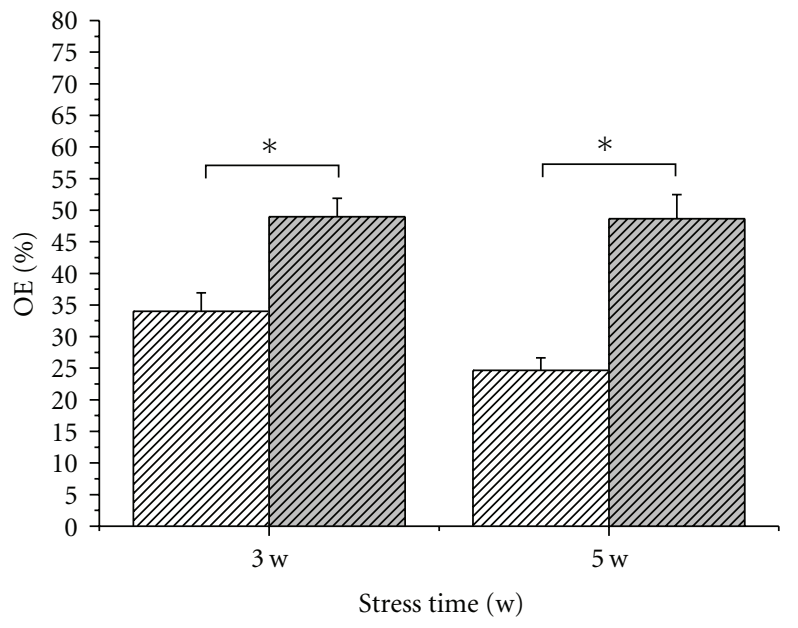

WIIA PS group

WIIA CON group

(a)

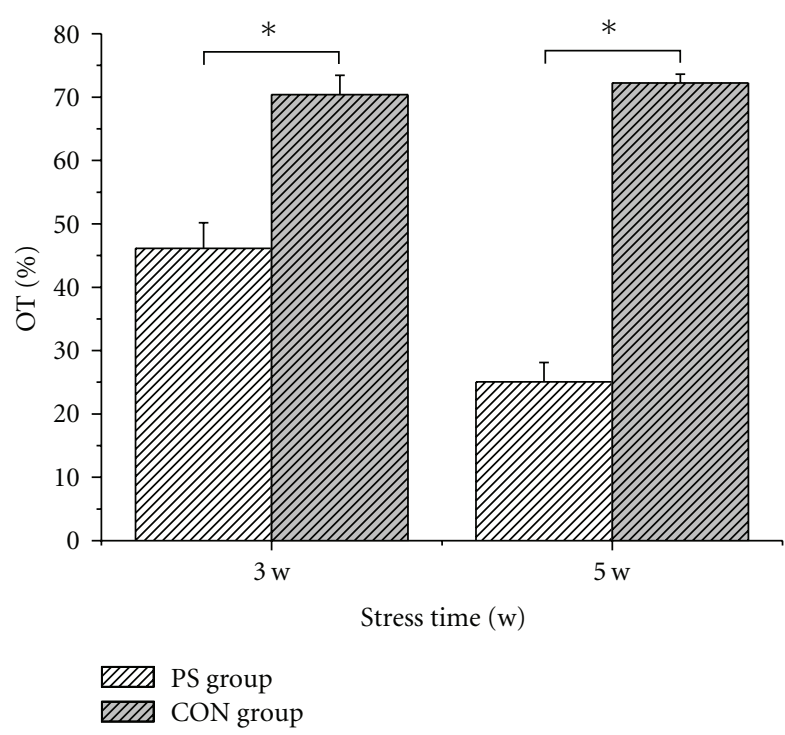

(b)

Figure 3: Measurement of rat behaviors in the elevated plus-maze test. Comparison of the percentage of open arm entries (OE\%) (a) and the percentage of time spent in the open arms (OT\%) (b) in the EPM test among groups. Data were expressed as $\bar{X} \pm \mathrm{SE}$ and analyzed in eight rats from each group $\left({ }^{*} P<.05\right.$, PS group versus CON group).

as shown in Figure 3(b), percentages of time spent in open arms (OT\%) significantly decreased from $70.39 \pm 3.03 \%$ in the control group to $46.14 \pm 4.05 \%$ in the PS group after three weeks of psychological stimulation and from $72.25 \pm$ $1.35 \%$ in the control group to $25.05 \pm 3.07 \%$ in PS group after five weeks $(P<.05)$, indicating that the rats in PS group were mentally under tension.

Next, we examined the ultrastructures of the masticatory muscles from both the control and PS groups after three and five weeks of psychological stimulation. As shown in Figure 4, the ultrastructures of the masticatory muscle 
cells of rats in the control and PS groups showed evenly distributed muscular nuclei under the sarcolemma around muscle fibers and no signs of hyperplasia, swelling, or pyknosis (Panels 1, 4, and 7). Myofibril and myotome of the PS rats were as normal as the rats in the control group. The light I bands and dark A bands of myotomes in the PS rats were intact and in the right position (Panels 4 and 7). Swollen mitochondria with cristae loss and reduction of matrix density were found in the $62.5 \%$ (5 of eight samples) and $87.5 \%$ (7 of eight samples) of the masticatory muscles samples of 3-w and 5-w PS rats, respectively, (as marked by black arrows in Panel 5 and 8). These ultrastructural observations are consistent with the possibility that the mitochondria from muscles of stressed mice may have altered permeability characteristics. Moreover, dramatic vacuolar changes in mitochondria appeared in PS rats after five weeks of stimulation (as marked by white arrows in Panel 8), which approximately were found in all samples $(87.5 \%$, 7 of eight samples). In contrast, there were nearly no swollen/abnormal mitochondria or vacuolar changes being observed in unstressed animal, which also gave the proof that the mitochondria changes observed in the PS rats has no relationship with the TEM sample processing.

The ultrastructure of capillaries in all control specimens were normal. The cytoplasm contained ribosomes, small strands of rough endoplasmic reticulum, and occasional small mitochondria. The outer membrane of each capillary was generally smooth, whereas their inner surfaces were often more convoluted with small cytoplasmic processes projecting into the lumen (Panel 3). The outer capillary wall was enclosed within a basal lamina approximately $20 \mathrm{~nm}$ in thickness. Portions of pericytes were often attached to the outer aspect of capillary basal laminae. The morphology of most capillaries in each specimen of 3-w PS rat was essentially normal, and the endothelial cell thickness appeared to be similar to that of the control specimens. But 2 of eight samples showed a sign of electron density decrease in the capillary cells (as marked by black arrow in Panel 6). As to the capillaries ultrastructure of the 5-w PS rats, cytoplasm of some capillary cells appeared to be more electron-lucent than those in control specimens (as marked by black arrows in Panel 9), with an increase in vesicle number and membrane ruffling. Some places of the capillary basal laminae seemed accidented or even discontinuous (as marked by white arrows in Panel 9), which appeared in about $75 \%$ of all samples (6 of eight samples) and meant local hypoxia and increased vascular permeability to some extent. These results implied that the ultrastructures of masticatory muscle cells were distinctly affected in PS rats after three and five weeks of psychological stimulation.

With regard to energy metabolism in the masticatory muscles, $\mathrm{Na}^{+}-\mathrm{K}^{+}$ATPase activity in the masticatory muscles decreased significantly from $7.60 \pm 0.50 \mu \mathrm{mol} \mathrm{Pi} / \mathrm{mg}$ protein/hour in the control group to $4.10 \pm 0.53 \mu \mathrm{mol}$ $\mathrm{Pi} / \mathrm{mg}$ protein/hour in the PS rats after three weeks of stimulation and even decreased from $7.15 \pm 0.35 \mu \mathrm{mol} \mathrm{Pi} / \mathrm{mg}$ protein/hour in the control group to $2.50 \pm 0.32 \mu \mathrm{mol}$ $\mathrm{Pi} / \mathrm{mg}$ protein/ hour in the PS group after five weeks of stimulation $(P<.05)$ (Figure 5(a)). Similarly, $\mathrm{Ca}^{2+}$-ATPase activity of the masticatory muscles decreased from $8.38 \pm$ 0.23 and $8.47 \pm 0.37 \mathrm{Pi} / \mathrm{mg}$ protein/hour in the control group to $3.82 \pm 0.58 \mu \mathrm{mol} \mathrm{Pi} / \mathrm{mg}$ protein/hour $(P<.05)$ and to $2.14 \pm 0.43 \mu \mathrm{mol} \mathrm{Pi} / \mathrm{mg}$ protein/hour $(P<.05)$ in the PS rats after three and five weeks of stimulation, respectively, (Figure 5(b)). By contrast, the activities of $\mathrm{LDH}$ and the contents of LD, respectively, significantly increased from $32.41 \pm 2.35(\mathrm{U} / \mathrm{g}$ protein $)$ and $0.67 \pm 0.10 \mathrm{mmol} / \mathrm{g}$ protein in the control group to $85.13 \pm 9.63$ (U/g protein) and 1.25 $\pm 0.19 \mathrm{mmol} / \mathrm{g}$ protein in the PS group after three weeks of stimulation $(P<.05)$ and even increased to $128.69 \pm$ 10.19 (U/g protein) and $1.97 \pm 0.33 \mathrm{mmol} / \mathrm{g}$ protein in the PS group after five weeks of stimulation $(P<.05)$ (Figures $5(c)$ and $5(d)$ ). There were also significant differences in the activities of $\mathrm{Na}^{+}-\mathrm{K}^{+}$ATPase, $\mathrm{Ca}^{2+}$-ATPase, and LDH and in the contents of LD between the three-week and five-week PS groups $(P<.05)$.

\section{Discussion}

To our knowledge, this is the first study to report significant changes in the ultrastructures and energy metabolism of the masticatory muscles of rats exposed to psychological stress.

The communication box is a well-established method for introducing psychological stress to animals. In this paradigm, the animals that do not undergo physical stress are able to perceive the responses of their neighboring animals that are exposed to physical stress, which is delivered through an electric foot shock. The intraspecies emotional communication then signals the nonstimulated rats to become anxious and can cause increased plasma level of stress hormones, such as corticosterone, in the experimental subjects [34]. In this paper, we successfully simulated a psychologically stressful environment using the communication box as indicated by the increased levels of cortisol and ACTH (Figure 2) and the decreased OT\% and OE\% in the EPM test (Figure 3) after three and five weeks of psychological stimulations.

It has been demonstrated that psychological stress is accompanied by regional modification of muscle morphology, possibly due to differential gene expressions or accessibility to hormones in certain regions of the masticatory muscle [22]. The present study observed subtle but significant changes in the ultrastructure of the rat masticatory muscles under psychological stress. Transmission electron microscopy demonstrated swollen mitochondria with cristae loss and reduction of matrix density in rats under psychological stress for three weeks (Figure 4, Panels 2, 5, and 8) and severe vacuolar changes in the mitochondria of the rats under a longer period of psychological stress (Figure 4, Panels 3, 7, and 9), suggesting that there were likely some important changes in mitochondrial function secondary to psychological stress. Therefore, we further investigated energy metabolism in the muscle, which is primarily associated with mitochondrial function.

$\mathrm{Na}^{+}-\mathrm{K}^{+}$ATPase and $\mathrm{Ca}^{2+}$-ATPase are key factors that help to maintain and modulate mitochondrial function. 


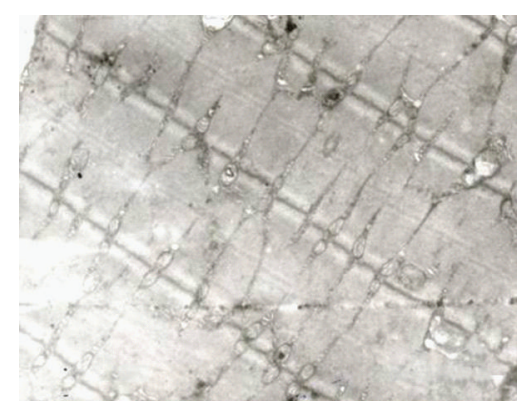

(1)

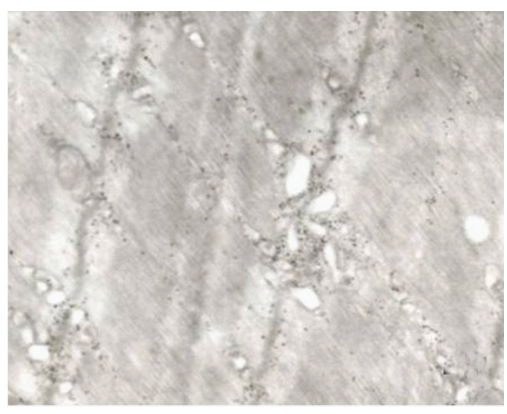

(4)

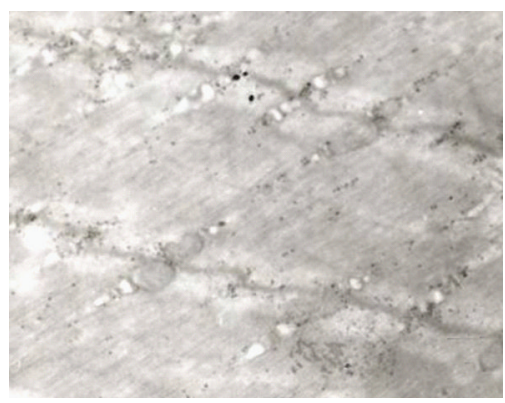

(7)

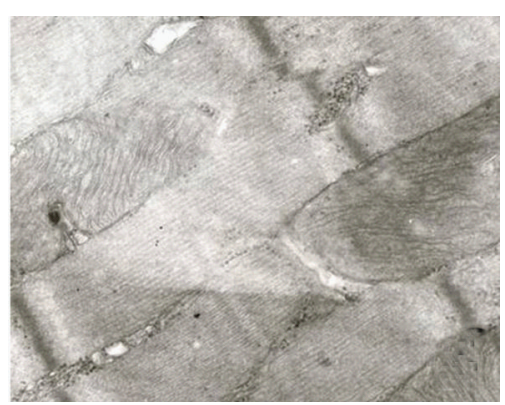

(2)

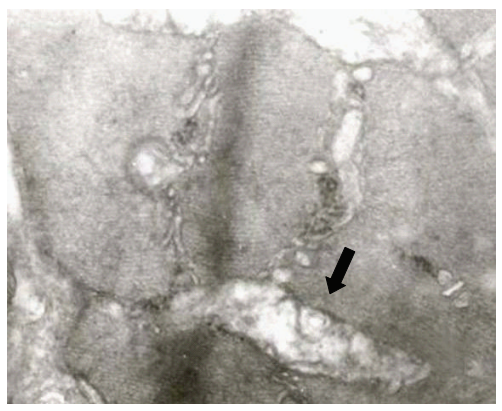

(5)

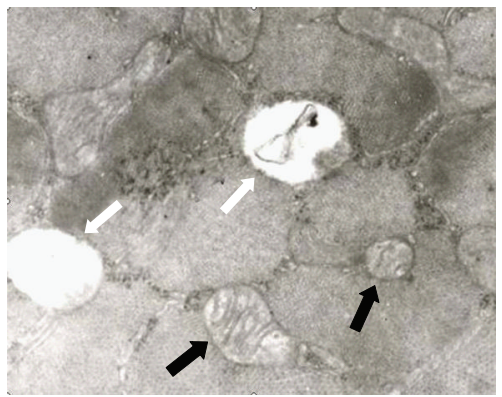

(8)

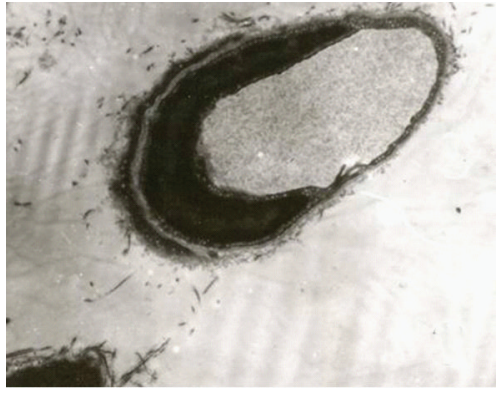

(3)

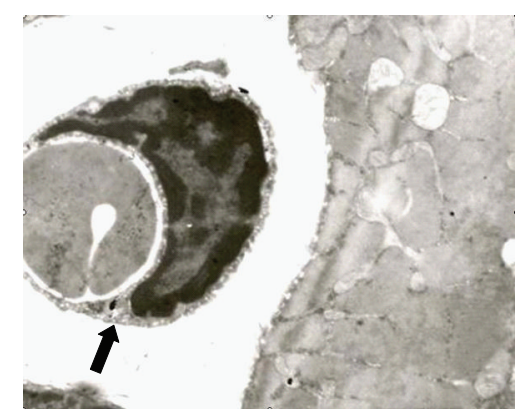

(6)

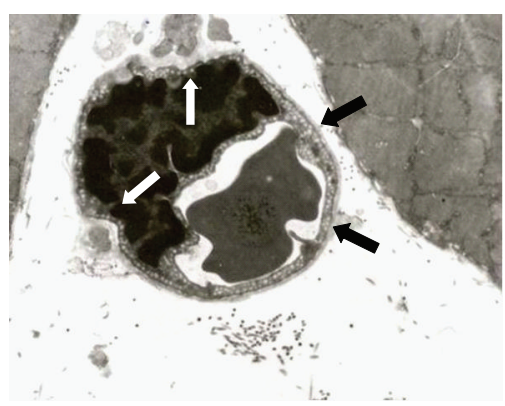

(9)

Figure 4: Electron microscopy of masticatory muscles. Shown are the ultrastructures of the masticatory muscles observed by transmission electron microscopy at a magnification of 8000X (panel 1, 2, 4, 5, 7, and 8) and 25000X (panel 3, 6, and 9). Panels 1, 2, and 3 refer to the control group (CON). Panels 4, 5, and 6 refer to the psychological group (PS) after three weeks of stimulation. Panels 7, 8, and 9 refer to PS after five weeks of stimulation. Swollen mitochondria with cristae loss and reduction of matrix density were found in the masticatory muscles of PS rats after three weeks or five weeks of stimulation, as marked by black arrows in Panel 5 and 8 . Dramatic vacuolar changes in mitochondria appeared in PS rats after five weeks of stimulation, as marked by white arrows in Panel 8. The ultrastructure of capillaries in both control (Panel 3) and 3-w PS specimens (Panel 6) were generally normal, except a few signs of electron density decrease in the capillary cells, as marked by black arrow in Panel 6. Cytoplasm of some capillary cells of 5-w PS rats appeared to be more electron-lucent than those in control specimens, as marked by black arrows in Panel 9. Some places of the capillary basal laminae became accidented, with significantly increased exudation around, as marked by white arrows in Panel 9.

Increased ATPase activity accelerates the rate of metabolism of muscle cells and improves muscle exercise capacity. Decreased ATPase activity is one of the signs of cell damage, which is often associated with hypoxia, acidic metabolites, or free radical formation [35]. Increases in lactate, concomitant with glycogen breakdown, are the result of an increased need by the muscle for an anaerobic energy supply. LDH activity level plays an important role in the removal of lactic acid and maintaining normal $\mathrm{pH}$ in the tissues, and it is an important enzyme in anaerobic oxidation [36]. We found that psychological stress results in decreased $\mathrm{Na}^{+}-\mathrm{K}^{+}$ATPase and $\mathrm{Ca}^{2+}$-ATPase activity, increased LDH activity, and elevated LD content in masticatory muscles, indicating a decrease in aerobic glycolysis and hypoxia or ischemia of the muscles. With increased exposure to psychological stress, the aerobic metabolism of the masticatory muscle decreased (Figures 5(a) and 5(b)), while anaerobic metabolism increased (Figures 5(c) and 5(d)), implying correlations between mitochondrial ultrastructure and energy metabolism in rat masticatory muscles. Similar results have been observed in the 

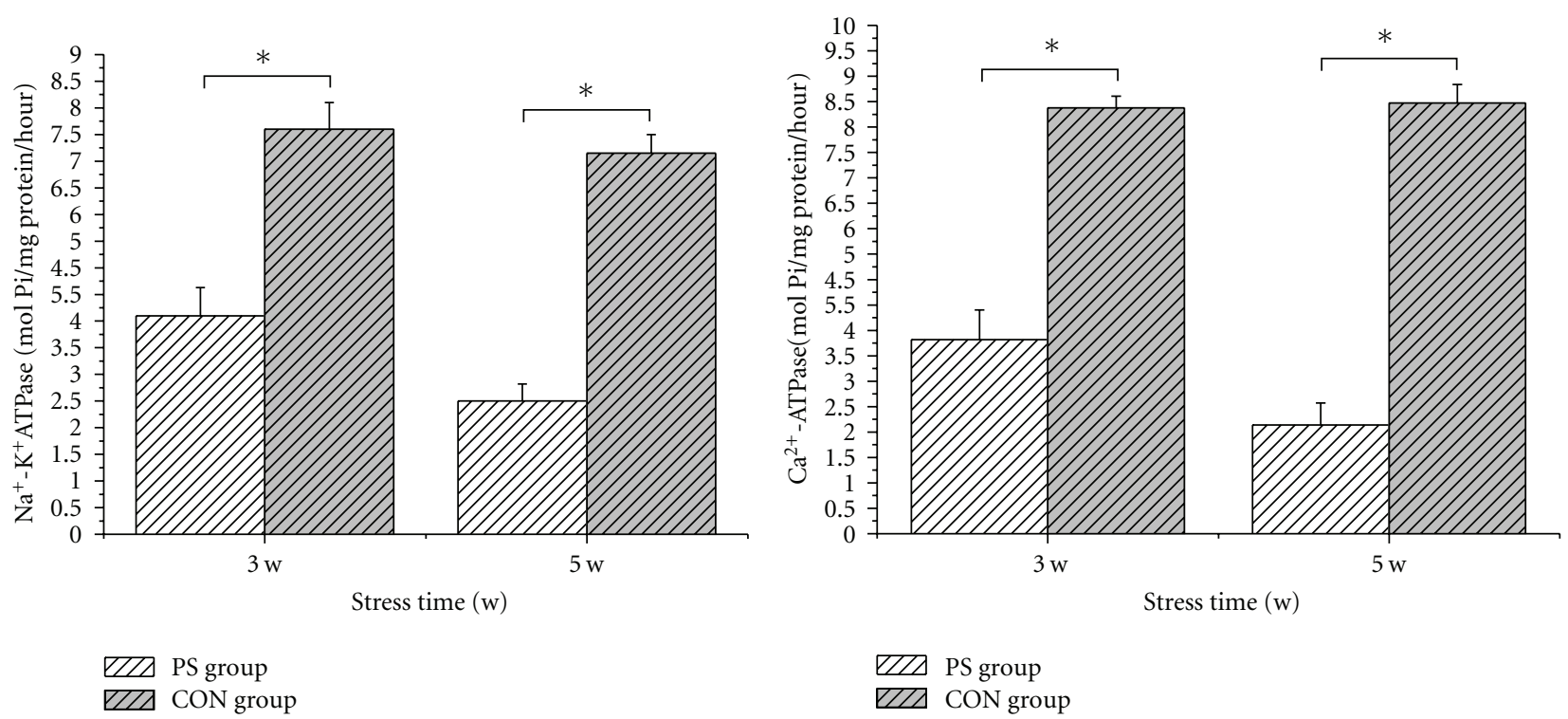

EZI CON group

(a)
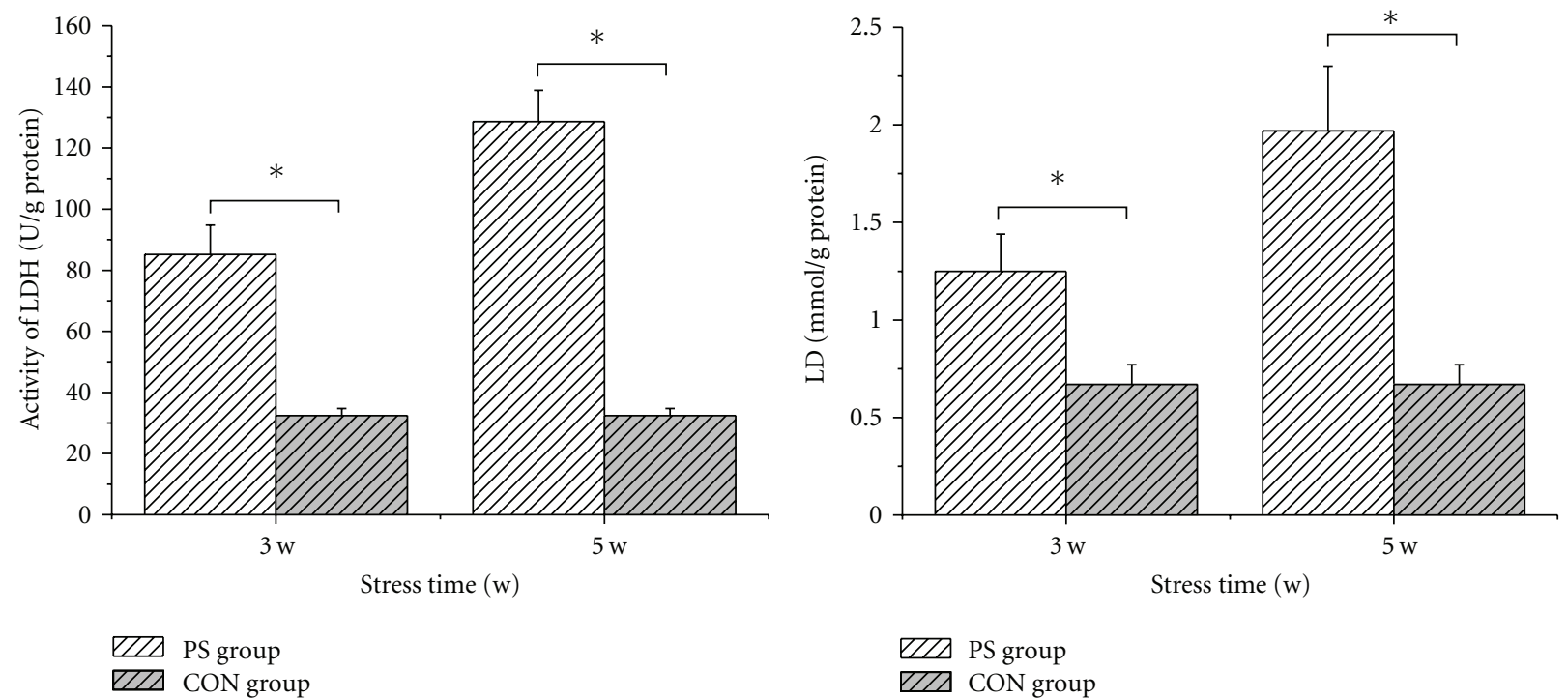

(c)

(d)

Figure 5: The activities of $\mathrm{Na}^{+}-\mathrm{K}^{+}$ATPase, $\mathrm{Ca}^{2+}$-ATPase, and lactate dehydrogenase (LDH) and the contents of lactic acid (LD) of the masticatory muscle. Shown are the activities of the enzymes $\mathrm{Na}^{+}-\mathrm{K}^{+}$ATPase (a), $\mathrm{Ca}^{2+}$-ATPase (b), and Lactate dehydrogenase (c) as well as the contents of lactic acid (d) in the masticatory muscles from the control (CON) and psychological (PS) groups detected by colorimetric methods. Data were expressed as $\bar{X} \pm \mathrm{SE}$ and analyzed in eight rats from each group ( ${ }^{*} P<.05$, PS group versus CON group).

atrial cardiomyocytes of rats exposed to audiogenic stress for $6 \mathrm{~h}$ [37].

Psychological stress, such as anxiety and tension, has been reported to increase myoelectricity $[3,18]$ and excessive activities of the masticatory muscle, including bruxism [21]. It has been suggested that the hemodynamics of jaw muscles are susceptible to mental stress via their regulation by the sympathetic nervous system (SNS) [23, 24], which is generally beneficial in the acute state of stress as it helps the organism to cope with changing environmental conditions and to reobtain homeostasis. Hidaka et al. observed a decrease in oxygen saturation of muscle blood around the onset of mental stress [23]. This is consistent with our observations by energy metabolism analysis showing decrease in aerobic glycolysis and hypoxia or ischemia of the muscles by mental stress. Widegren et al. reported that the vascular response of skeletal muscle in individuals under mental stress is mainly a vasodilation, rather than a vasoconstriction [38], which is consistent with the widened space around the capillaries and increased vascular permeability observed in this paper by TEM.

Emotional stressors induce masseter muscle contractions [39-41] that may be based on a pathway and may, in part, be mediated as follows: sensory inputs occur via the 
thalamus (activation of the amygdale, especially basolateral complex). Efferents to various CNS systems, included muscular contraction, via ventral amygdalofugal pathway to the brainstem, specifically the trigeminal motor neurons [42]. The effect on cranial nerve nuclei and components induced by emotional stress (cranial nerve V, masseters; cranial nerve VII, muscles of facial expression) also lead to mouth opening and jaw movements that are supposedly facial expressions of fear [16]. There also has been theorized that compression of the blood vessels caused by muscle contraction could result in the release of local myogenic and metabolic vasodilatation factors. Therefore, adequate blood flow is usually guaranteed in the working muscles, as the sympathetic vasoconstriction is antagonized and overridden by the powerful local vasodilator action of metabolites released by the contracting muscles. The imbalance between these actions, the excessive muscle contraction/metabolites, and an insufficient vasodilation induced by the SNS may create ischemia, which might be responsible for the decreased aerobic metabolism and increased vascular permeability of masseter capillaries.

Clinically, these results emphasize the importance of environmental influences on the emotional state, and these influences could in turn affect the ultrastructure and energy metabolism of masticatory muscles. Whether these alterations are reversible or reducible needs to be further investigated with countermeasures, such as drugs, physiotherapy, and psychological stress-free environments.

\section{Conclusions}

Psychological stress can cause mitochondrial injuries and hyperemia of the masticatory muscle capillaries in rats. Further, such stress can result in dramatic alterations in the energy metabolism of the masticatory muscles. Following prolonged psychological stress, these changes become more severe. Thus, this might reflect a possible mechanism for the pathogenesis of masticatory muscle dysfunction.

\section{Acknowledgments}

This study was financially supported by the National Natural Science Foundation of China (no. 30672326). The authors are deeply thankful to Professor Jian Liu of the Fourth Military Medical University of China (Xi'an, China) for his earnest guidance. Y. J. Chen and F. Huang contributed equally to this work.

\section{References}

[1] I. Uhac, Z. Kovac, M. Valentic-Peruzovic, M. Juretic, L. J. Moro, and R. Gržic, "The influence of war stress on the prevalence of signs and symptoms of temporomandibular disorders," Journal of Oral Rehabilitation, vol. 30, no. 2, pp. 211-217, 2003.

[2] S. M. Rao and A. G. Glaros, "Electromyographic correlates of experimentally induced stress in diurnal bruxists and normals," Journal of Dental Research, vol. 58, no. 9, pp. 18721878, 1979.
[3] C.-M. Tsai, S.-L. Chou, E. N. Gale, and W. D. Mccall Jr., "Human masticatory muscle activity and jaw position under experimental stress," Journal of Oral Rehabilitation, vol. 29, no. 1, pp. 44-51, 2002.

[4] D. M. Laskin, "Etiology of the pain-dysfunction syndrome," The Journal of the American Dental Association, vol. 79, no. 1, pp. 147-153, 1969.

[5] L. L. Schwartz, "Pain associated with the temporomandibular joint," The Journal of the American Dental Association, vol. 51, no. 4, pp. 394-397, 1955.

[6] W. L. Kydd, "Psychosomatic aspects of temporomandibular joint dysfunction," The Journal of the American Dental Association, vol. 59, no. 1, pp. 31-44, 1959.

[7] N. Alamoudi, "Correlation between oral parafunction and temporomandibular disorders and emotional status among Saudi children," Journal of Clinical Pediatric Dentistry, vol. 26, no. 1, pp. 71-80, 2001.

[8] T. List, K. Wahlund, and B. Larsson, "Psychosocial functioning and dental factors in adolescents with temporomandibular disorders: a case-control study," Journal of Orofacial Pain, vol. 15, no. 3, pp. 218-227, 2001.

[9] E. L. Schiffman, J. R. Fricton, and D. Haley, "The relationship of occlusion, parafunctional habits and recent life events to mandibular dysfunction in a non-patient population," Journal of Oral Rehabilitation, vol. 19, no. 3, pp. 201-223, 1992.

[10] J. F. Casanova-Rosado, C. E. Medina-Solís, A. A. VallejosSánchez, A. J. Casanova-Rosado, B. Hernández-Prado, and L. Ávila-Burgos, "Prevalence and associated factors for temporomandibular disorders in a group of Mexican adolescents and youth adults," Clinical Oral Investigations, vol. 10, no. 1, pp. 42-49, 2006.

[11] A. U. J. Yap, S. F. Dworkin, E. K. Chua, T. List, K. B. C. Tan, and H. H. Tan, "Prevalence of temporomandibular disorder subtypes, psychologic distress, and psychosocial dysfunction in Asian patients," Journal of Orofacial Pain, vol. 17, no. 1, pp. 21-28, 2003.

[12] J. A. Turner, L. Mancl, and L. A. Aaron, "Brief cognitivebehavioral therapy for temporomandibular disorder pain: effects on daily electronic outcome and process measures," Pain, vol. 117, no. 3, pp. 377-387, 2005.

[13] D. Townsend, R. A. Nicholson, L. Buenaver, F. Bush, and S. Gramling, "Use of a habit reversal treatment for temporomandibular pain in a minimal therapist contact format," Journal of Behavior Therapy and Experimental Psychiatry, vol. 32, no. 4, pp. 221-239, 2002.

[14] M. A. Gardea, R. J. Gatchel, and K. D. Mishra, "Long-term efficacy of biobehavioral treatment of temporomandibular disorders," Journal of Behavioral Medicine, vol. 24, no. 4, pp. 341-359, 2001.

[15] T. I. Suvinen, P. C. Reade, P. Kemppainen, M. Könönen, and S. F. Dworkin, "Review of aetiological concepts of temporomandibular pain disorders: towards a biopsychosocial model for integration of physical disorder factors with psychological and psychosocial illness impact factors," European Journal of Pain, vol. 9, no. 6, pp. 613-633, 2005.

[16] J. C. Türp, A. Hugger, P. Nilges et al., "Recommendations for the standardized evaluation and classification of painful temporomandibular disorders: an update," Schmerz, vol. 20, no. 6, pp. 481-489, 2006.

[17] H. Flor, N. Birbaumer, W. Schulte, and R. Roos, "Stressrelated electromyographic responses in patients with chronic temporomandibular pain," Pain, vol. 46, no. 2, pp. 145-152, 1991. 
[18] R. A. Nicholson, D. R. Townsend, and S. E. Gramling, "Influence of a scheduled-waiting task on EMG reactivity and oral habits among facial pain patients and no-pain controls," Applied Psychophysiology Biofeedback, vol. 25, no. 4, pp. 203 219, 2000

[19] S. Ruf, F. Cecere, J. Kupfer, and H. Pancherz, "Stress-induced changes in the functional electromyographic activity of the masticatory muscles," Acta Odontologica Scandinavica, vol. 55, no. 1, pp. 44-48, 1997.

[20] P. Richardin, A. Westphal, M. Divry, and G. Didier, "Influence of stress and occlusal interference on the EMG activity of some masticatory muscles during a single mastication cycle," Journal of oral rehabilitation, vol. 22, no. 10, pp. 775-780, 1995.

[21] V. P. Rosales, K. Ikeda, K. Hizaki, T. Naruo, S.-I. Nozoe, and G. Ito, "Emotional stress and brux-like activity of the masseter muscle in rats," European Journal of Orthodontics, vol. 24, no. 1, pp. 107-117, 2002.

[22] E. Cvetko and I. Eržen, "Effect of chronic uncontrollable stress on myosin heavy chain expression in rat masseter muscle," Pflugers Archiv European Journal of Physiology, vol. 442, no. 6, supplement 1, pp. R147-R149, 2001.

[23] O. Hidaka, M. Yanagi, and K. Takada, "Changes in masseteric hemodynamics time-related to mental stress," Journal of Dental Research, vol. 83, no. 2, pp. 185-190, 2004.

[24] O. Hidaka, M. Yanagi, and K. Takada, "Mental stress-induced physiological changes in the human masseter muscle," Journal of Dental Research, vol. 83, no. 3, pp. 227-231, 2004.

[25] K. Ayada, M. Watanabe, and Y. Endo, "Elevation of histidine decarboxylase activity in skeletal muscles and stomach in mice by stress and exercise," American Journal of Physiology, vol. 279, no. 6, pp. R2042-R2047, 2000.

[26] B. Lennartsson, "Number and distribution of muscle spindles in the masticatory muscles of the rat," Journal of Anatomy, vol. 130, no. 2, pp. 279-288, 1980.

[27] U. Proske, A. K. Wise, and J. E. Gregory, "The role of muscle receptors in the detection of movements," Progress in Neurobiology, vol. 60, no. 1, pp. 85-96, 2000.

[28] T. Yamamoto and T. Une, "Animal models of psychiatric disorder and their validity-from the perspective of behavioral pharmacology," Nippon Yakurigaku Zasshi, vol. 120, no. 3, pp. 173-180, 2002.

[29] S. Pellow, P. Chopin, S. E. File, and M. Briley, "Validation of open: closed arm entries in an elevated plus-maze as a measure of anxiety in the rat," Journal of Neuroscience Methods, vol. 14, no. 3, pp. 149-167, 1985.

[30] R. G. Lister, "The use of a plus-maze to measure anxiety in the mouse," Psychopharmacology, vol. 92, no. 2, pp. 180-185, 1987.

[31] G. Biała and M. Kruk, "Amphetamine-induced anxiety-related behavior in animal models," Pharmacological Reports, vol. 59, no. 6, pp. 636-644, 2007.

[32] M. Rabbani, S. E. Sajjadi, and A. Mohammadi, "Evaluation of the anxiolytic effect of Nepeta persica Boiss. in mice," Evidence-based Complementary and Alternative Medicine, vol. 5, no. 2, pp. 181-186, 2008.

[33] M. M. Bradford, "A rapid and sensitive method for the quantitation of microgram quantities of protein utilizing the principle of protein dye binding," Analytical Biochemistry, vol. 72, no. 1-2, pp. 248-254, 1976.

[34] M. Ishikawa, C. Hara, S. Ohdo, and N. Ogawa, "Plasma corticosterone response of rats with sociopsychological stress in the communication box," Physiology and Behavior, vol. 52, no. 3, pp. 475-480, 1992.
[35] D. G. Allen, "Skeletal muscle function: role of ionic changes in fatigue, damage and disease," Clinical and Experimental Pharmacology and Physiology, vol. 31, no. 8, pp. 485-493, 2004.

[36] J. M. Lawler, S. K. Powers, T. Visser, H. Van Dijk, M. J. Kordus, and L. L. Ji, "Acute exercise and skeletal muscle antioxidant and metabolic enzymes: effects of fiber type and age," American Journal of Physiology, vol. 265, no. 6, pp. R1344-R1350, 1993.

[37] A. Paparelli, A. Pellegrini, P. Lenzi, M. Gesi, and P. Soldani, "Ultrastructural changes in atrial tissue of young and aged rats submitted to acute noise stress," Journal of Submicroscopic Cytology and Pathology, vol. 27, no. 1, pp. 137-142, 1995.

[38] U. Widegren, R. C. Hickner, L. Jorfeldt, and J. Henriksson, "Muscle blood flow response to mental stress and adrenaline infusion in man: microdialysis ethanol technique compared to ${ }^{133} \mathrm{Xe}$ clearance and venous occlusion plethysmography," Clinical Physiology and Functional Imaging, vol. 30, no. 2, pp. 152-161, 2010.

[39] S. Landgren and K. A. Olsson, "The effect of electrical stimulation in the hypothalamus on the monosynaptic jaw closing and the disynaptic jaw opening reflexes in the cat," Experimental Brain Research, vol. 39, no. 4, pp. 389-400, 1980.

[40] Y. Yamada, K. Uchida, and T. Sato, "Mandibular movement trajectories and masticatory muscle activities in the rabbit in the sleep and wake states," Dentistry in Japan, vol. 27, no. 1, pp. 35-39, 1990.

[41] P. Sitthisomwong, S. Weiner, L. Levin, S. Reisman, and A. Siegel, "The effect of a cholecystokinin agonist on masseter muscle activity in the cat," Journal of Dental Research, vol. 79, no. 10, pp. 1823-1828, 2000.

[42] V. P. Rosales, K. Ikeda, K. Hizaki, T. Naruo, S.-I. Nozoe, and G. Ito, "Emotional stress and brux-like activity of the masseter muscle in rats," European Journal of Orthodontics, vol. 24, no. 1, pp. 107-117, 2002. 


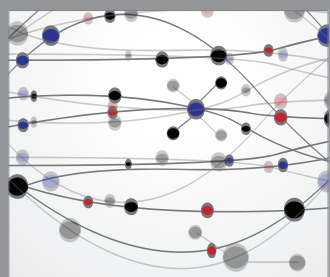

The Scientific World Journal
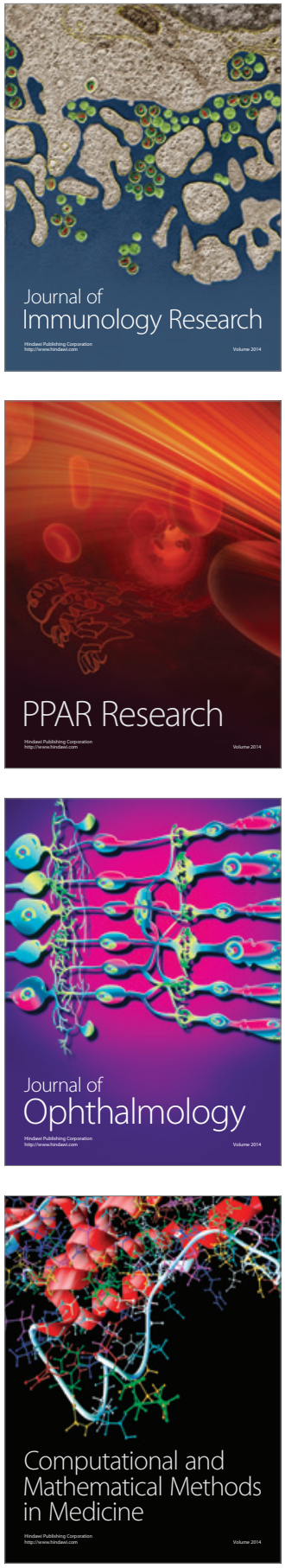

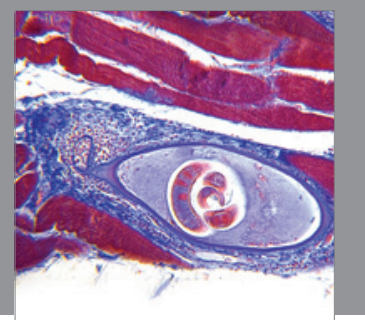

Gastroenterology

Research and Practice
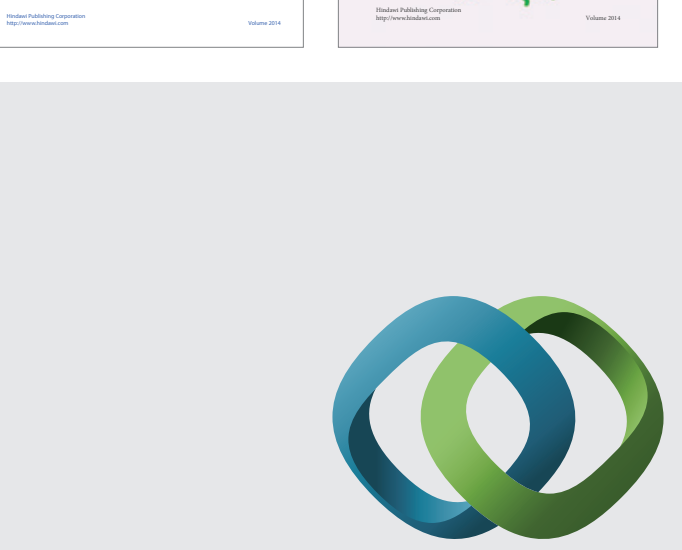

\section{Hindawi}

Submit your manuscripts at

http://www.hindawi.com
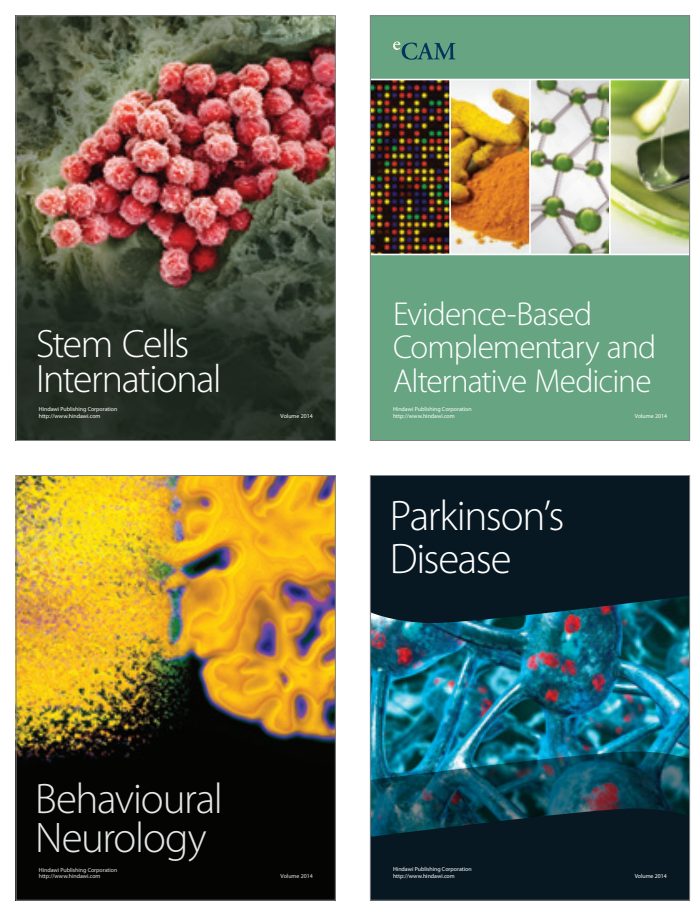

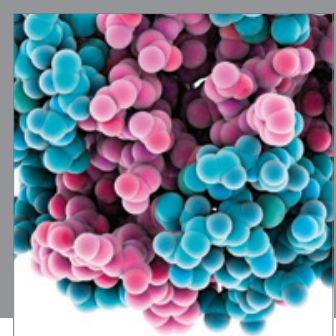

Journal of
Diabetes Research

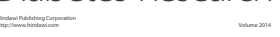

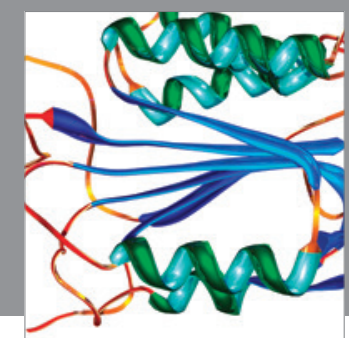

Disease Markers
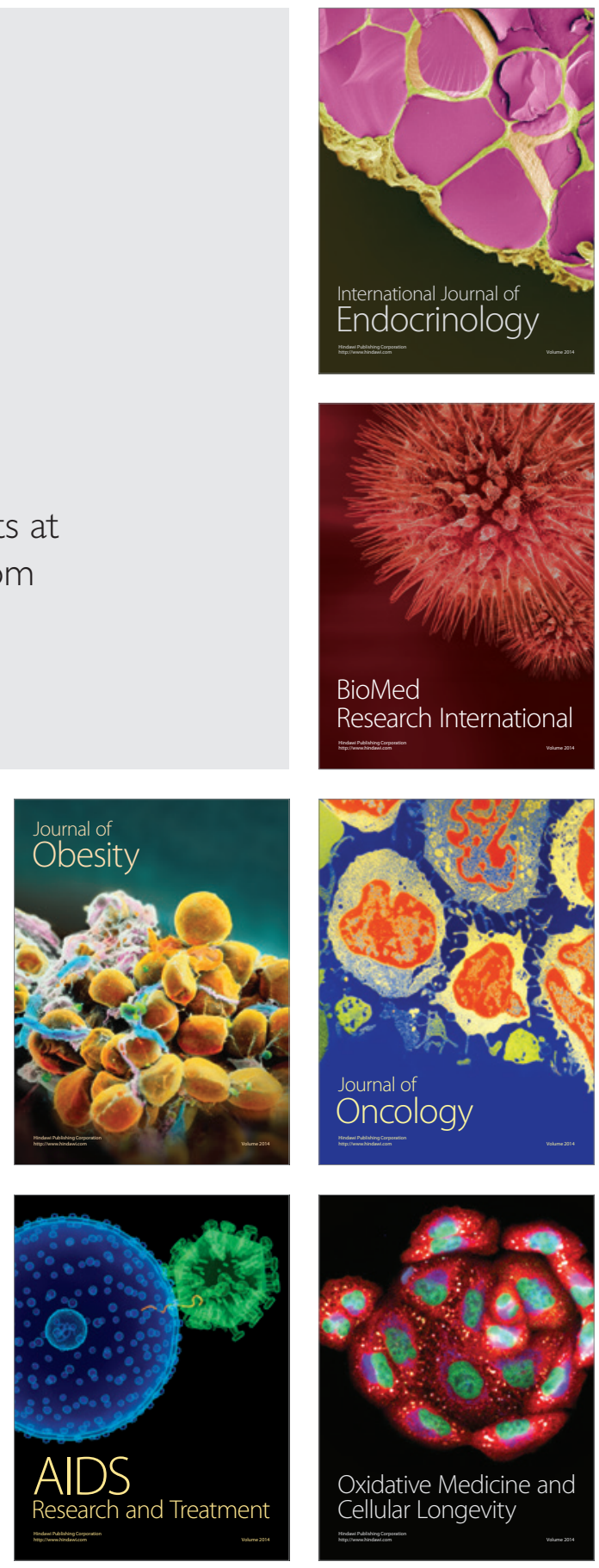